\title{
Transverse Momentum Distributions of Hadrons Produced in Pb-Pb Collisions at LHC Energy $\sqrt{\mathbf{s}_{\mathrm{NN}}}=2.76 \mathbf{T e V}$
}

\author{
Saeed Uddin, Inam-ul Bashir, and Riyaz Ahmed Bhat \\ Department of Physics, Jamia Millia Islamia (Central University), New Delhi 110025, India \\ Correspondence should be addressed to Saeed Uddin; saeed_jmi@yahoo.co.in
}

Received 12 August 2014; Revised 21 October 2014; Accepted 28 October 2014

Academic Editor: Edward Sarkisyan-Grinbaum

Copyright (C) 2015 Saeed Uddin et al. This is an open access article distributed under the Creative Commons Attribution License, which permits unrestricted use, distribution, and reproduction in any medium, provided the original work is properly cited. The publication of this article was funded by SCOAP $^{3}$.

\begin{abstract}
The transverse momentum spectra of several types of hadrons, $p, \bar{p}, K^{+}, K^{-}, K_{s}^{0}, \Lambda, \Omega, \bar{\Omega}, \Xi^{-}$, and $\bar{\Xi}$ produced in most central $\mathrm{Pb}-\mathrm{Pb}$ collisions at LHC energy $\sqrt{\mathbf{s}_{\mathrm{NN}}}=2.76 \mathrm{TeV}$ have been studied at midrapidity $(|y|<0.5)$ using an earlier proposed unified statistical thermal freeze-out model. The calculated results are found to be in good agreement with the experimental data measured by the ALICE experiment at LHC. The model calculation fits provide the thermal freeze-out conditions in terms of the temperature and collective flow effect parameters for different particle species. Interestingly the model parameter fits to the experimental data reveal stronger collective flow in the system and lesser freeze-out temperatures of the different particle species as compared to $\mathrm{Au}-\mathrm{Au}$ collisions at RHIC. The strong increase of the collective flow appears to be a consequence of the increasing particle density at LHC. The model used incorporates a longitudinal as well as transverse hydrodynamic flow. The chemical potential has been assumed to be nearly equal to zero for the bulk of the matter owing to high degree of nuclear transparency effect at such collision energies. The contributions from heavier decay resonances are also taken into account.
\end{abstract}

\section{Introduction}

The study of identified particle spectra in heavy-ion collisions at ultrarelativistic energies is an important tool to investigate the properties of the strongly interacting system created in such collisions. The study also helps us to learn about the final state distribution of baryon numbers among various particle species at the thermochemical freeze-out after the collision which is initially carried by the nucleons only [1].

Within the framework of the statistical model, it is assumed that a hot and dense fireball is formed over an extended region for a brief period of time $(\sim \mathrm{a}$ few $\mathrm{fm} / \mathrm{c})$ after the initial collision and it undergoes collective expansion leading to a decrease in its temperature and finally to the hadronization. After the hot fireball formed in such collisions, which initially has a very high density of partons (i.e., quarks and gluons), hadronizes, the hadrons keep rescattering with each other and continue to build up collective expansion. Consequently, the matter becomes dilute and the average distance between hadrons exceeds the range of the strong interactions. At this point of time, all scattering processes stop and the hadrons decouple; that is, a freeze out occurs [2].

The hadronic abundance freeze-out (i.e., the chemical freeze-out) occurs earlier when the rates for inelastic processes, in which secondary hadrons are produced or the hadrons change their identity (via strangeness exchange process, etc.), become too small to keep up with the expansion. Since the corresponding inelastic cross sections are only a small fraction of the total cross section at lower (thermal) energies, the inelastic processes stop well before the elastic ones, leading to an earlier chemical freeze-out for the hadron abundances. Finally at a later stage the hadrons completely decouple from each other such that even the elastic processes also come to a stop. Consequently, the momentum spectra get frozen in time and a thermal (or hydrodynamical) freeze-out occurs. Thus chemicalfreeze-outprecedes thermal or kinetic freeze-out [3].

Transverse momentum $\left(p_{T}\right)$ distributions of identified hadrons are the most common tools used to study the dynamics of high energy collisions. 
The identified particle spectra provide information, about both the temperature of the system and the collective flow at the time of thermal freeze-out. Collective flow depends on the internal pressure gradients created in the collision and is addressed by hydrodynamic models [4-6]. These effects are species-dependent. The produced hadrons are believed to carry information about the collision dynamics and the subsequent space-time evolution of the system.

Hence an accurate measurement of the transverse momentum distributions of identified hadrons along with the rapidity spectra is essential for the understanding of the dynamics and the properties of the created matter up to the final thermal or hydrodynamical freeze-out in case of collective flow [7].

It has been shown earlier [2] that this model successfully simultaneously explains the rapidity and transverse momentum distributions of hadrons and their ratios in $\mathrm{Au}-\mathrm{Au}$ collisions at highest RHIC energy of $\sqrt{\mathbf{s}_{\mathrm{NN}}}=200 \mathrm{GeV}$. In this paper, we briefly describe the model and use it to reproduce the transverse momentum distributions of hadrons produced in $\mathrm{Pb}-\mathrm{Pb}$ collisions at $\sqrt{\mathbf{s}_{\mathrm{NN}}}=2.76 \mathrm{TeV}$.

\section{Model}

In order to obtain the particle spectra in the overall rest frame of the hadronic fireball in our model we first define the invariant cross-section for given hadronic specie in the local rest frame of a hadronic fluid element. Since the invariant cross section will have the same value in all Lorentz frames [8], we can thus write

$$
E \frac{d^{3} N}{d^{3} p}=E^{\prime} \frac{d^{3} N}{d^{3} p^{\prime}}
$$

where $E\left(E^{\prime}\right)$ is the energy of the particle and $p\left(p^{\prime}\right)$ is the momentum. The primed quantities on the RHS refer to the invariant spectra of given hadronic specie in the rest frame of the local hadronic fluid element, while the unprimed quantities on the LHS refer to the invariant spectra of the same hadronic specie in the overall rest frame of the hadronic fireball. The occupation number distribution of the hadrons in the momentum space follows the distribution function:

$$
E^{\prime} \frac{d^{3} N}{d^{3} p^{\prime}} \sim \frac{E^{\prime}}{e^{\left(\left(E^{\prime}-\mu\right) / T\right)} \pm 1},
$$

where $(+)$ sign and $(-)$ sign are for fermions and bosons, respectively, and $\mu$ is the chemical potential of the given hadronic specie. For the temperatures under consideration and the large masses of hadrons it is safe to work with Boltzman distribution.

In recent works $[7,9]$ it has been clearly shown that there is a strong evidence of increasing baryon chemical potential, $\mu_{B}$, along the collision axis in the RHIC experiments. In view of this fact we write the expression for chemical potential as $\mu_{B}=\mathbf{a}+\mathbf{b} y_{0}^{2}[7,9,10]$, where $\mathbf{y}_{\mathbf{0}}$ is the rapidity of the expanding hadronic fluid element. Here $\mathbf{a}$ and $\mathbf{b}$ are the two model parameters which can be fixed by fitting the experimental data. In the model the value of a essentially defines the baryon chemical potential in the central region of the bulk hadronic matter formed, while $\mathbf{b}$ determines the rate of increase of baryon chemical potential along the (longitudinal) rapidity axis with $\mathbf{y}_{\mathbf{0}}$. In case of very high degree of nuclear transparency the values of $\mathbf{a}$ and $\mathbf{b}$ will tend to vanish for the bulk of the matter. Further it is assumed that [7] the rapidity of the expanding hadronic fluid element $y_{0} \alpha z$ or $y_{0}=\xi z$, where $z$ is the longitudinal coordinate of the hadronic fluid element and $\xi$ is a proportionality constant. The above conditions also ensure that, under the transformation $z \rightarrow-z$, we will have $y_{0} \rightarrow-y_{0}$, thereby preserving the symmetry of the hadronic fluid flow about $z=0$ along the rapidity axis in the centre of mass frame of the colliding nuclei. This leads to an expression for the longitudinal velocity component of the hadronic fluid element:

$$
\beta_{z}(z)=1-\frac{2}{\exp (2 \xi z)+1}=\tanh \left(y_{0}\right) .
$$

The transverse velocity component of the hadronic fireball, $\beta_{T}$ is assumed to vary with the transverse coordinate $r$ in accordance with the blast wave model as $\beta_{T}(r)=\beta_{T}^{s}(r / R)^{n}$ [11], where $n$ is an index which fixes the profile of $\beta_{T}(r)$ in the transverse direction and $\beta_{T}^{s}$ is the hadronic fluid surface transverse expansion velocity and is fixed in the model by using the parameterization $\beta_{T}^{s}=\beta_{T}^{0} \sqrt{1-\beta_{z}^{2}}$ [7]. This relation is also required to ensure that the net velocity $\beta$ of any fluid element must satisfy $\beta=\sqrt{\beta_{T}^{2}+\beta_{z}^{2}}<1$. We also parameterize $R$, that is, the transverse radius of fireball as $R=r_{0} \exp \left(-z^{2} / \sigma^{2}\right)$ where $\sigma$ fixes the width of the matter distribution in the transverse direction [7, 9] and $z$, as described above, is the longitudinal coordinate of hadronic fluid element. This is required as the colliding nuclei when passing through each other may still feel some drag thus resulting only in a partial transparency. Consequently, the collision axis will be populated by an extended hadronic matter rapidly moving away from each other with its transverse size decreasing rapidly following a Gaussian distribution along the $z$-axis.

In our analysis, the contributions of various heavier hadronic resonances $[10,12]$ which decay after the thermal freeze-out of the hadronic matter has occurred are also taken into account. The invariant spectrum of a given decay product of a given parent hadron in the local rest frame of a hadronic fluid element is written as $[7,10,12]$

$$
E^{\prime} \frac{d^{3} N^{\text {decay }}}{d^{3} p^{\prime}}=\frac{1}{2 p^{\prime}}\left\{\frac{m_{h}}{p^{*}}\right\} \int_{E_{-}}^{E_{+}} d E_{h} E_{h}\left\{\frac{d^{3} N_{h}}{d^{3} p_{h}}\right\},
$$

where the subscript $h$ stands for the decaying (parent) hadron. The two body decay kinematics gives the product hadron's momentum and energy in the "rest frame of the decaying hadron" as $p^{*}=\left(E^{* 2}-m^{2}\right)^{1 / 2}$ and $E^{*}=\left(m_{h}^{2}-\right.$ $\left.m_{j}^{2}+m^{2}\right) / 2 m_{h}$, where $m_{j}$ indicates the mass of the other decay hadron produced along with the first one. The limits of integration are $E_{ \pm}=\left\{m_{h} / m^{2}\right\}\left\{E^{\prime} E^{*} \pm p^{\prime} p^{*}\right\}$. The $E^{\prime}\left(E_{h}\right)$ and $p^{\prime}\left(p_{h}\right)$ are, respectively, the product (decaying parent) 


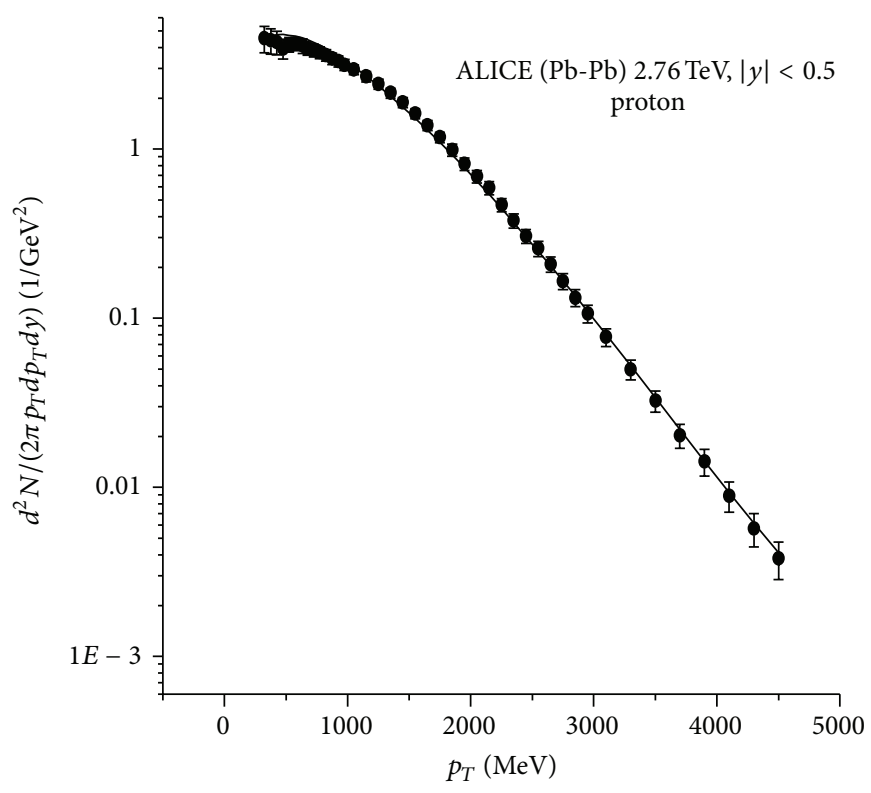

(a)

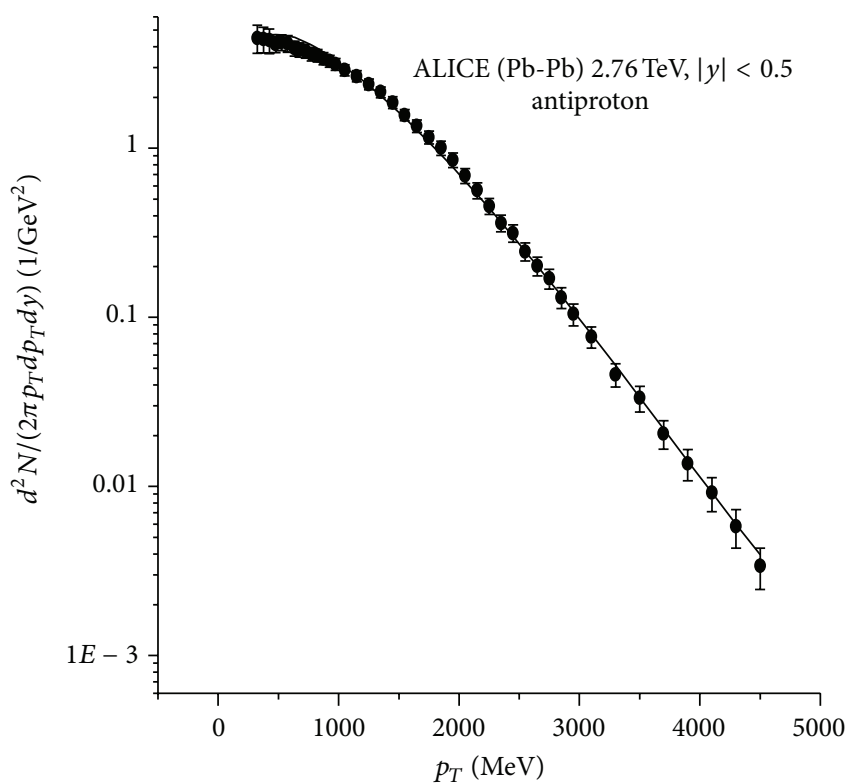

(b)

Figure 1: Transverse momentum spectra of protons (a) and antiprotons (b) for the centrality class (0-5)\%.

hadron's energy and momentum in the local rest frame of the hadronic fluid element. A Boltzmann type distribution for the massive decaying hadron in the local rest frame of the hadronic fluid element leads to the following final expression for the invariant cross section of the product hadron:

$$
\begin{aligned}
& E^{\prime} \frac{d^{3} N}{d^{3} p^{\prime}} \\
& =\frac{1}{2 p^{\prime}}\left\{\frac{m_{h}}{p^{*}}\right\} \lambda_{h} g_{h} e^{-\alpha \theta E^{\prime} E^{*}} \\
& \quad \times\left\{\frac{\alpha}{\theta}\left[E^{\prime} E^{*} \sinh \left(\alpha \theta p^{\prime} p^{*}\right)-p^{\prime} p^{*} \cosh \left(\alpha \theta p^{\prime} p^{*}\right)\right]\right. \\
& \left.+T^{2} \sinh \left(\alpha \theta p^{\prime} p^{*}\right)\right\},
\end{aligned}
$$

where $\alpha$ and $\theta$ are given by $m_{h} / m^{2}$ and $1 / T$, respectively.

\section{Results and Discussions}

We employ the minimum $x^{2} /$ DoF method to fit the experimental data. We find that the model calculations results (shown by solid curves in all the cases) fit the experimental data quite well (shown by filled circles in all the cases). The experimental data are taken from the ALICE Collaboration for $\mathrm{Pb}-\mathrm{Pb}$ collisions at $\sqrt{\mathbf{s}_{\mathrm{NN}}}=2.76 \mathrm{TeV}$ [13-15]. We have shown the (statistical + systematic) errors in all the cases.

Over a fairly large $p_{T}$ range the hydrodynamical calculations show an approximate exponential behavior, whereas the tails of measured spectra show a significant deviation in the slope beyond $5 \mathrm{GeV}$ at LHC. At RHIC this transition from exponential behavior takes place at $p_{T} \gtrsim 3 \mathrm{GeV}$. The fraction of hadrons with very large $p_{T}$ ( $\geq 3 \mathrm{GeV}$ at RHIC and $\geq 5 \mathrm{GeV}$ at LHC) is however small. We have considered the (maximum) $p_{T}$ range up to $5 \mathrm{GeV}$ in the present analysis. It is because that the statistical hydrodynamic calculations cannot describe the hadron spectra at such large transverse momenta. The hadrons detected in this region are essentially formed by the partons which are result of the hard processes. These originate from the direct fragmentation of high-energy partons of the colliding beams and therefore are not able to thermalize through the process of multiple collisions [16]. We therefore turn to softer hadrons which are assumed to be reasonably thermalized and form the bulk of the secondary matter produced.

The applicability region of hydrodynamics at LHC is therefore predicted to be for $p_{T} \leq 4-5 \mathrm{GeV}$ depending on the particle's mass. This range is wider than at RHIC [17]. The transverse momentum distributions are found to be sensitive to the values of the thermal/kinetic freeze-out temperature $T$ and the transverse flow parameter $\beta_{T}^{0}$, whereas it is found to be insensitive to the change in the values of $\sigma$ in our model. In our analysis we have therefore fixed the value of the parameter $\sigma=5.0$. This value essentially determines the size of the hadronic matter distributed along the $z$-axis and has a strong effect on the shape of the rapidity spectra of the particles. In our earlier analysis [2] of the RHIC data the value of $\sigma$ turned out to be nearly 4.2. However, it is expected to be large at the LHC energy. The insensitivity of the transverse momentum distribution to the parameter $\sigma$ has been tested and it is found that the minimum $x^{2} / \mathrm{DoF}$ for protons varies only from 0.611 to 0.614 if $\sigma$ is varied from 4.0 to 6.0. We have taken the values of $\mathbf{a}$ and $\mathbf{b}$ both to be zero for all the hadrons under the assumption of a baryon symmetric matter expected to be formed under the condition of a high degree of nuclear transparency in the nucleus-nucleus collisions at LHC energy 


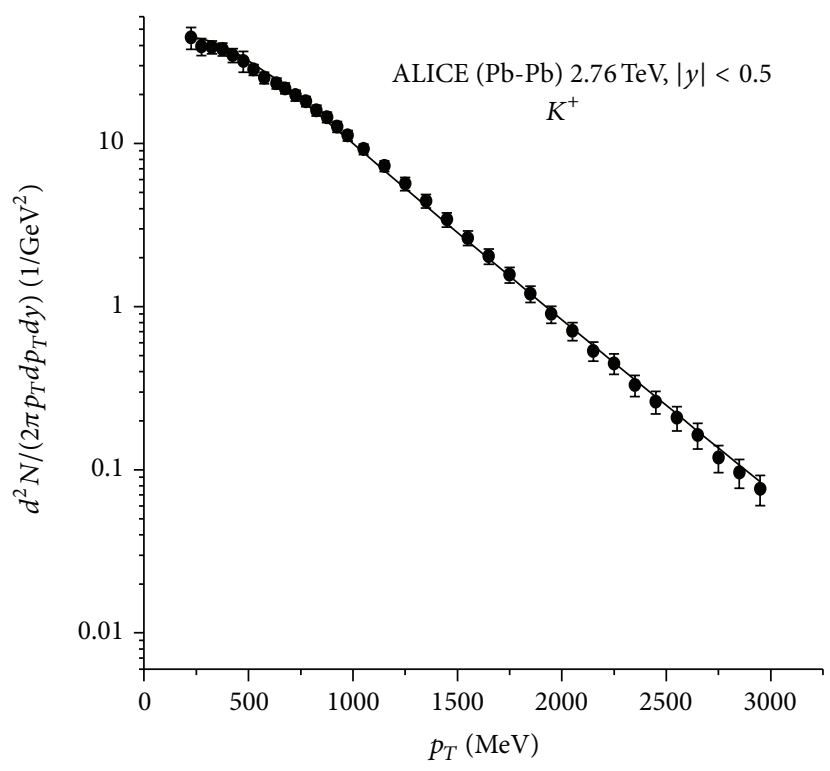

(a)

FIGURE 2: Transverse momentum spectra of $K^{+}$

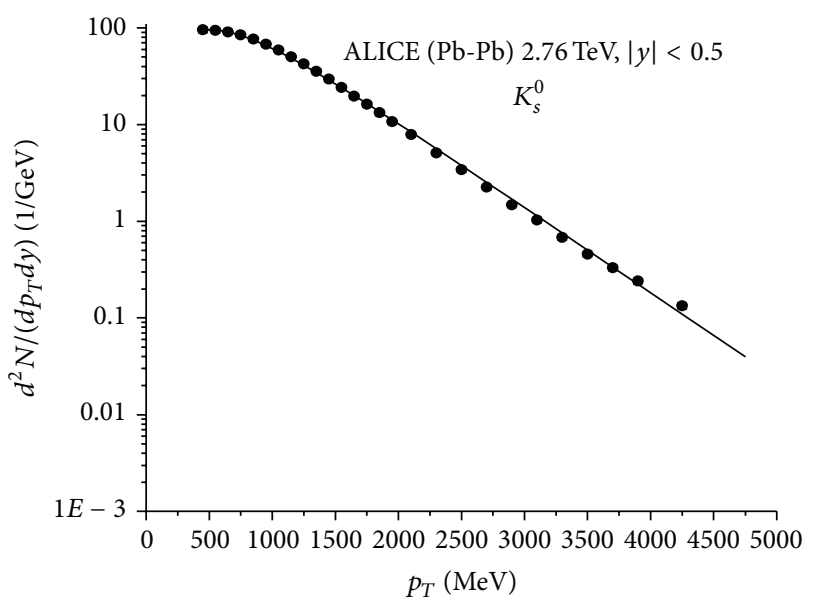

FIGURE 3: Transverse momentum spectra of $K_{s}^{0}$ for the centrality class $(0-5) \%$.

that is, an ideal Bjorken picture. Unlike the previous works we have in our present analysis treated the index parameter $n$ as a free parameter. The values of the parameters, $T, \beta_{T}^{0}$ and $n$, at freeze-out are determined by obtaining a best fit to a given hadron's transverse momentum spectrum. The value of $\xi=1$ is fixed for all the hadrons studied in this paper. The theoretical fits for the transverse momentum spectra of all the hadrons have been normalized at the first data point (i.e., at the lowest $p_{T}$ ) to facilitate a proper comparison with the experimental data set.

In Figure 1, we have shown the transverse momentum spectra of protons and antiprotons. The values of

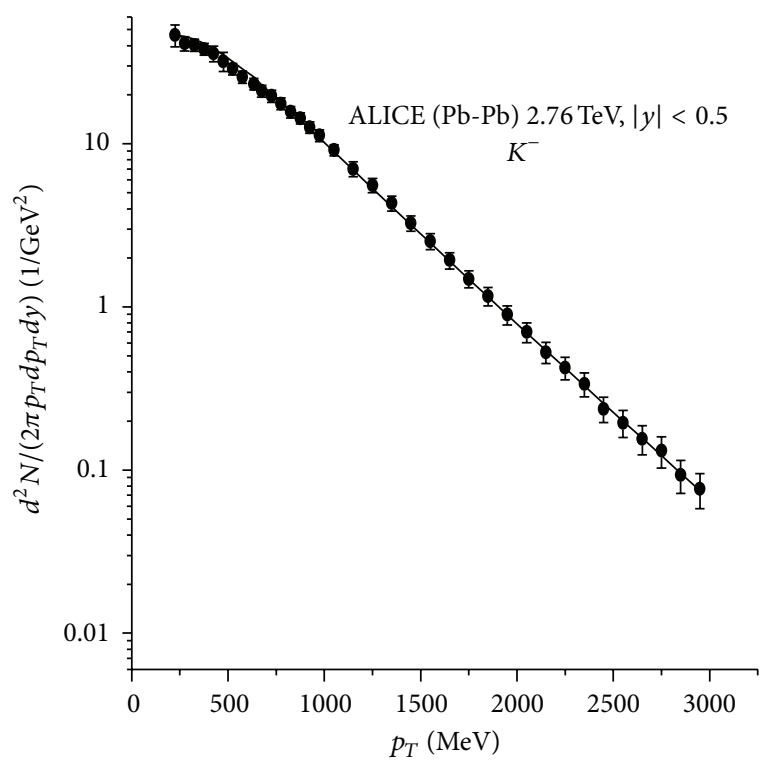

(b)

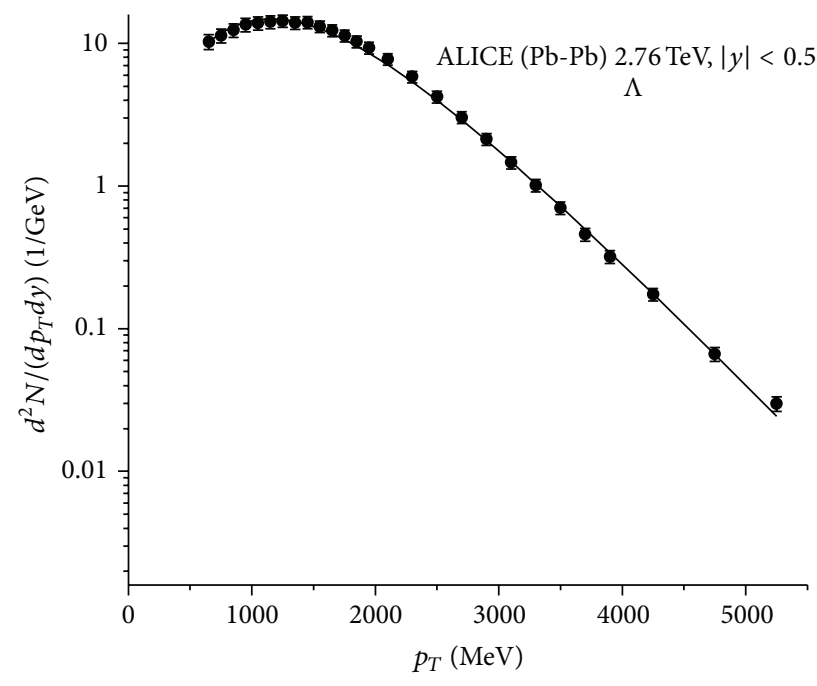

Figure 4: Transverse momentum spectra of lambda $\Lambda$ for the centrality class $(0-5) \%$.

the thermal/kinetic freeze-out temperature $T$, the transverse flow parameter $\beta_{T}^{0}$, and the index parameter $n$ for protons as well as antiprotons are found to be same, that is, $102 \mathrm{MeV}$, 0.88 , and 1.40 , respectively, with a minimum $x^{2} /$ DoF of 0.61 for protons and 0.55 for antiprotons. The same values of the freeze-out parameters for protons and antiprotons indicate a simultaneous freeze-out of these particles in the dense hadronic medium.

The transverse momentum spectra for $K^{+}$and $K^{-}$shown in Figure 2 gives the value of $\left(T, \beta_{T}^{0}\right.$, and $\left.n\right)$ as $(103 \mathrm{MeV}$, 0.89 , and 1.80) for Kaons and (105 MeV, 0.88 , and 1.80) for anti-Kaons. The minimum $x^{2} / \mathrm{DoF}$ for both the two cases 


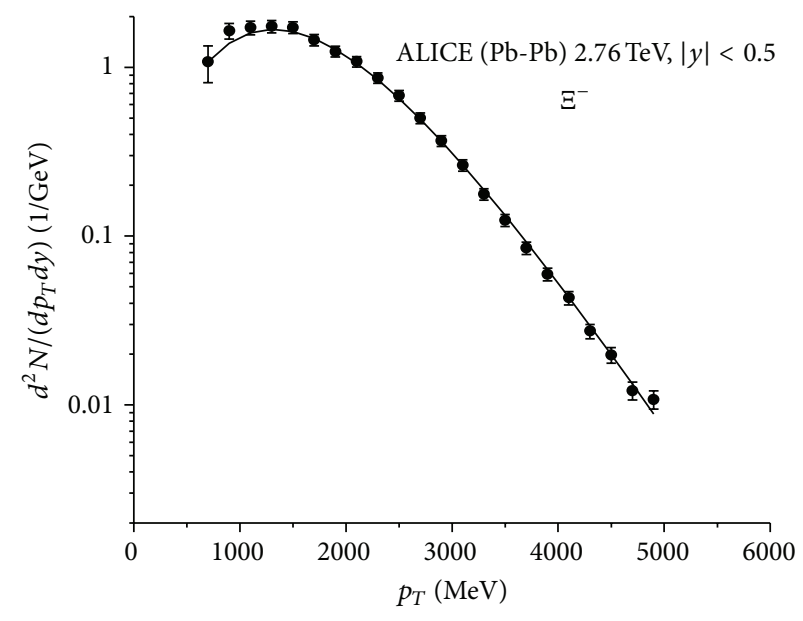

(a)

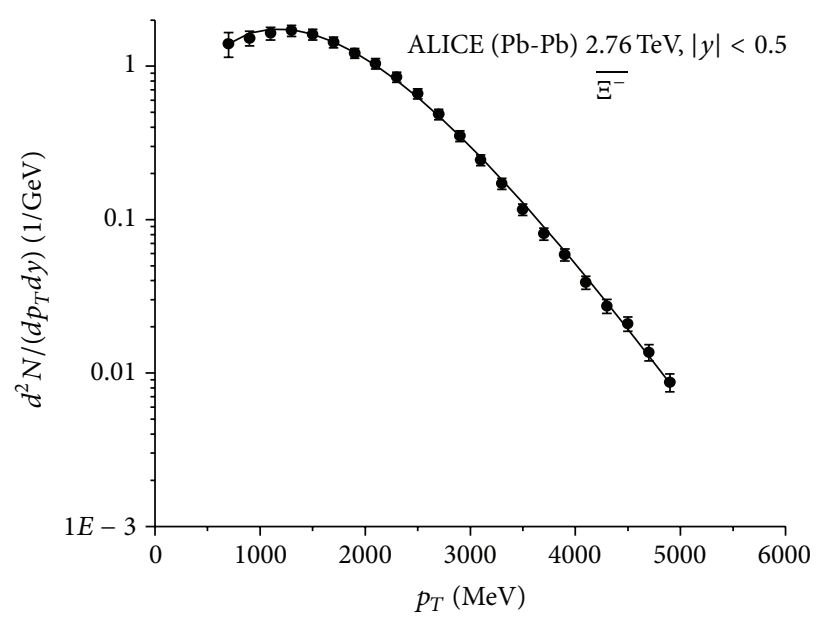

(b)

FigurE 5: Transverse momentum spectra of $\Xi^{-}$(a) and $\overline{\Xi^{-}}$(b) for the centrality class $(0-10) \%$.

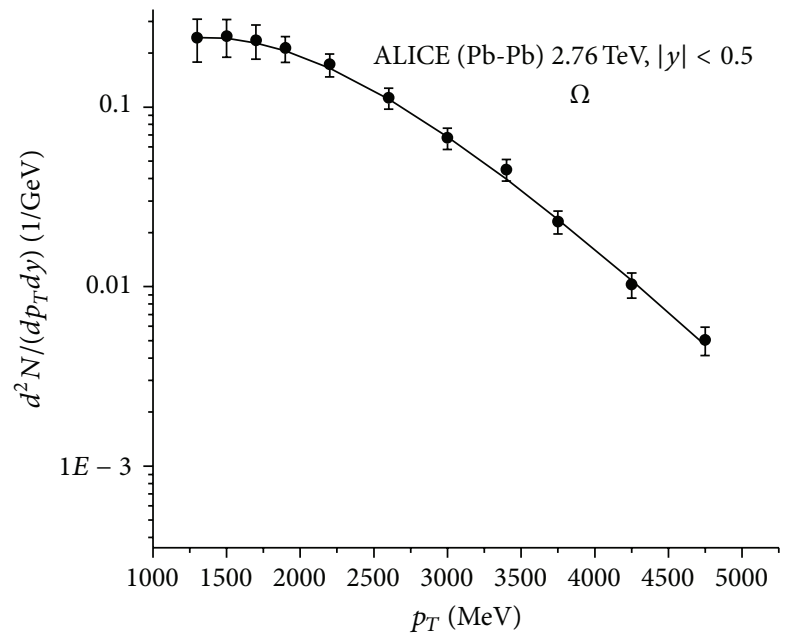

(a)

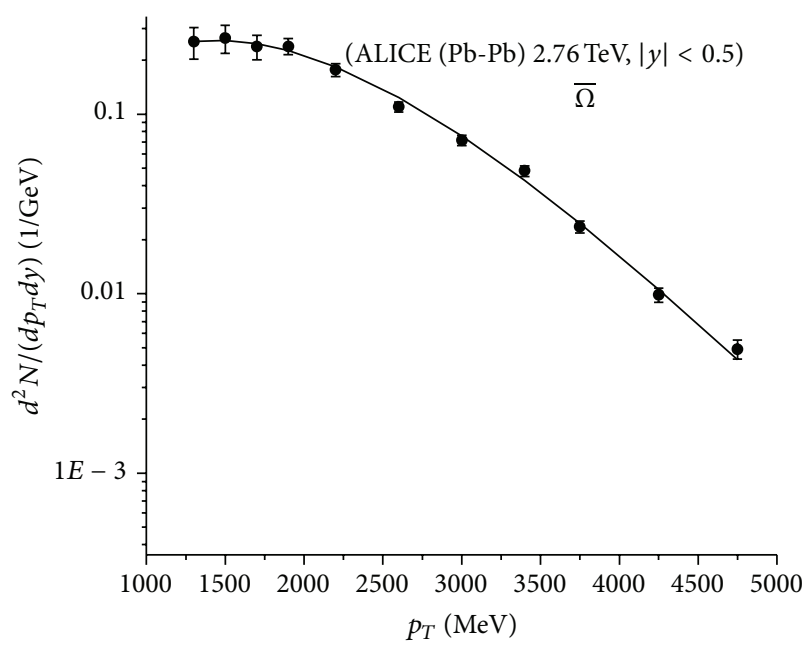

(b)

FIGURE 6: Transverse momentum spectra of $\Omega(\mathrm{a})$ and $\bar{\Omega}$ (b) for the centrality class $(0-10) \%$.

turns out to be 0.34 . The almost similar freeze-out parameters obtained for protons, antiprotons, Kaons, and anti-Kaons indicate a near simultaneous freeze-out of these particles.

The transverse momentum spectrum of neutral Kaon, that is, $K_{s}^{0}$, is shown in Figure 3. The values of $T, \beta_{T}^{0}$, and $n$ obtained from the spectra of $K_{s}^{0}$ are, respectively, $125 \mathrm{MeV}$, 0.84 , and 1.61 with the minimum $x^{2} / \mathrm{DoF}=1.70$. The $K_{s}^{0}$ shows a larger thermal freeze-out temperature than the charged Kaons indicating its earlier freeze-out than $K^{ \pm}$.

The transverse momentum spectra of hyperons (i.e., $\Lambda$, $\Xi^{-}, \overline{\Xi^{-}}, \Omega$ and $\bar{\Omega}$ ) are shown in Figures 4,5 , and 6 . The spectrum of $\Lambda$ gives the values of $T, \beta_{T}^{0}$, and $n$ as $127 \mathrm{MeV}$, 0.84 , and 1.06 , respectively, with a minimum $x^{2} / \mathrm{DoF}=$ 0.52 . These values for $\Xi^{-}$are found to be $133 \mathrm{MeV}, 0.81$, and 0.90 while for $\bar{\Xi}^{-}$these are $149 \mathrm{MeV}, 0.80$, and 1.25 , respectively. The parameters for $\Omega$ and $\bar{\Omega}$ are $(155 \mathrm{MeV}$,
0.77 , and 1.22$)$ and ( $154 \mathrm{MeV}, 0.77$, and 1.23$)$. The minimum $x^{2} /$ DoF for $\Xi^{-}$and $\bar{\Xi}^{-}$are 0.38 and 0.50 , whereas for $\Omega$ and $\bar{\Omega}$ the minimum $x^{2} /$ DoF are 0.10 and 0.20 , respectively. The relatively smaller values of minimum $x^{2} / \mathrm{DoF}$ for $\Omega s$ are due to larger experimental error bars.

The values of the thermal/kinetic freeze-out temperature $T$, the transverse flow parameter $\beta_{T}^{0}$, and the index parameter $n$ for all the hadrons studied in this paper are again presented in Table 1 to facilitate a proper comparison.

It is evident from Table 1 that the lighter particles, that is, (anti)protons and Kaons, exhibit a lower thermal freezeout temperature and a higher surface transverse expansion velocity compared to the heavy (multi)strange hyperons. The reason for this can be attributed to an early freezeout for the massive particles (hyperons) when the thermal temperature is high and the collective flow is in the early 


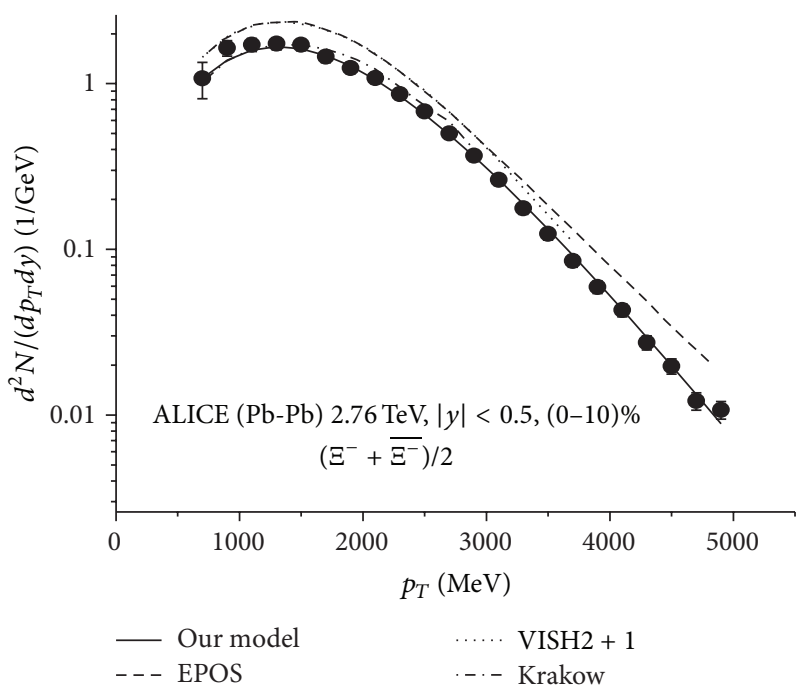

(a)



(b)

FIGURE 7: Transverse momentum spectra for $\left(\Xi^{-}+\overline{\Xi^{-}}\right) / 2$ (a) and $(\Omega+\bar{\Omega}) / 2$ (b) are compared to hydrodynamic models EPOS, VISH2 +1 , and KRAKOW for the $(0-10) \%$ most central $\mathrm{Pb}-\mathrm{Pb}$ collisions at $\sqrt{\mathbf{s}_{\mathrm{NN}}}=2.76 \mathrm{TeV}$.

TABLE 1: Freeze-out parameters of various hadrons obtained from their transverse momentum spectra.

\begin{tabular}{lcccc}
\hline Particle & $T(\mathrm{MeV})$ & $\beta_{T}^{0}$ & $n$ & $x^{2} / \mathrm{DoF}$ \\
\hline$p$ & 102 & 0.88 & 1.40 & 0.61 \\
$\bar{p}$ & 102 & 0.88 & 1.40 & 0.55 \\
$K^{+}$ & 103 & 0.89 & 1.80 & 0.34 \\
$K^{-}$ & 105 & 0.88 & 1.80 & 0.34 \\
$K_{s}^{0}$ & 125 & 0.84 & 1.61 & 1.70 \\
$\Lambda$ & 127 & 0.84 & 1.06 & 0.52 \\
$\Xi^{-}$ & 133 & 0.81 & 0.90 & 0.38 \\
$\Xi^{-}$ & 149 & 0.80 & 1.25 & 0.48 \\
$\Omega$ & 155 & 0.77 & 1.22 & 0.10 \\
$\bar{\Omega}$ & 154 & 0.77 & 1.23 & 0.20 \\
\hline
\end{tabular}

stage of development and consequently $\beta_{T}^{0}$ is small. The early freeze-out of these particles is due to their smaller crosssection with the hadronic matter. This can also be understood in terms of the mean free path, $\lambda$, of a particle in a thermal environment which is given by $\lambda=1 / v \rho$, where $v$ is the mean thermal cross section of the particle with the surrounding matter having density $\rho$. The transverse flow velocity profile index $n$, using the best fit method for the hadrons considered here, lies in the range 1.61-1.80 for the lightest particles considered here (i.e., Kaons), for (anti)protons it is 1.40 and for the heavier multistrange hyperon it lies in the range 0.901.25. Hence in general $n$ appears to be smaller for the heavier particles. Another observation is that, for the particles with lower $n$, the $p_{T}$ spectra show a shoulder-like shape at low transverse momenta.

A comparison with a similar fit to the RHIC data $[2,6]$ shows that for the most central collisions the flow velocity increases significantly at LHC reaching almost 0.9 and that the kinetic freeze-out temperature drops below the one at RHIC. For RHIC [2] the values of $T$ and $\beta_{T}^{0}$ were found to be in the range $163-188 \mathrm{MeV}$ and $0.58-0.67$, respectively.

We have also compared the results of some other model calculations like VISH2+1, HKM, and Krakow models [13, 18 ] with our model results in Figure 7 for the transverse momentum spectra of $\left(\Xi^{-}+\overline{\Xi^{-}}\right) / 2$ and $(\Omega+\bar{\Omega}) / 2$. The Krakow model results are available up to nearly $3 \mathrm{GeV}$ for $\left(\Xi^{-}+\overline{\Xi^{-}}\right) / 2$ and nearly $2.5 \mathrm{GeV}$ for $(\Omega+\bar{\Omega}) / 2$.

In the first case the KRAKOW model results are seen to fit the experimental data reasonably well but provides a bad fit for the second case, that is, $(\Omega+\bar{\Omega}) / 2$. The VISH $2+1$ and HKM describe the shape of these spectra somewhat better; however, the VISH $2+1$ overestimates the yield of $(\Omega+$ $\bar{\Omega}) / 2$ at larger $p_{T}$. Also it is found [18] that Krakow model overestimates the yield of protons up to $1.75 \mathrm{GeV}$. In contrast, our model successfully reproduces the shape of the transverse momentum distributions of these hadrons in a wider $p_{T}$ range up to $5 \mathrm{GeV}$.

\section{Conclusion}

The transverse momentum spectra of the hadrons $p, \bar{p}, K^{+}$, $K^{-}, K_{s}^{0}, \Lambda, \Omega, \bar{\Omega}, \Xi^{-}$and $\overline{\Xi^{-}}$are fitted quite well by using our model. The assumption of vanishing chemical potential at midrapidity shows the effects of almost complete transparency in $\mathrm{Pb}-\mathrm{Pb}$ collisions at LHC energy of $2.76 \mathrm{TeV}$. We also observe an earlier freeze-out of hyperons as compared to lighter mass particles, that is, Kaons and protons. The protons, antiprotons, Kaons, and anti-Kaons have similar freeze-out conditions, which indicate their near simultaneous freezeout from the dense hadronic medium. The larger values of $\beta_{T}^{0}$ at the LHC energy, as compared to those at RHIC, indicates a stronger flow effect present in the system at LHC. 
The spectra are compared with the predictions from some other hydrodynamic models also and it is found that a better fit is obtained by using our model covering a wider $p_{T}$ range up to $5 \mathrm{GeV}$.

\section{Conflict of Interests}

The authors declare that there is no conflict of interests regarding the publication of this paper.

\section{Acknowledgments}

Inam-ul Bashir is thankful to University Grants Commission for awarding him the Basic Scientific Research (BSR) Fellowship. Riyaz Ahmed Bhat is grateful to Council of Scientific and Industrial Research, New Delhi, for awarding him Senior Research Fellowship. Saeed Uddin is grateful to University Grants Commission for financial assistance.

\section{References}

[1] E. Kornas, "Energy dependence of proton and antiproton production in central $\mathrm{Pb}+\mathrm{Pb}$ collisions from NA49," The European Physical Journal C, vol. 49, pp. 293-296, 2007.

[2] S. Uddin, R. A. Bhat, I. ul Bashir, W. Bashir, and J. S. Ahmad, "Systematic of particle thermal freeze-out in a hadronic fireball at RHIC," In press, http://xxx.tau.ac.il/abs/1401.0324.

[3] U. W. Heinz, "Concepts of heavy-ion physics," http://arxiv.org/ abs/hep-ph/0407360.

[4] W. Broniowski and W. Florkowski, "Description of the RHIC $p_{\perp}$ spectra in a thermal model with expansion," Physical Review Letters, vol. 87, Article ID 272302, 2001.

[5] W. Broniowski and W. Florkowski, "Description of strange particle production in $\mathrm{Au}+\mathrm{Au}$ collisions of $\sqrt{\mathbf{s}_{\mathrm{NN}}}=130 \mathrm{GeV}$ in a single-freeze-out model," Physical Review C, vol. 65, Article ID 064905, 2002.

[6] D. Teaney, J. Lauret, and E. V. Shuryak, "Flow at the SPS and RHIC as a quark-gluon plasma signature," Physical Review Letters, vol. 86, no. 21, pp. 4783-4786, 2001.

[7] S. Uddin, J. S. Ahmad, W. Bashir, and R. Ahmad Bhat, "A unified approach towards describing rapidity and transverse momentum distributions in a thermal freeze-out model," Journal of Physics G, vol. 39, no. 1, Article ID 015012, 2012.

[8] S. Sarkar, H. Satz, and B. Sinha, The Physics of the Quark-Gluon Plasma, vol. 785 of Lecture Notes in Physics, Spinger, Berlin, Germany, 2010.

[9] F. Becattini, J. Cleymans, and J. Strumpfer, "Rapidity variation of thermal parameters at SPS and RHIC," http://arxiv .org/abs/0709.2599.

[10] S. Uddin, J. S. Ahmad, M. Ali, W. Bashir, R. A. Bhat, and M. F. Mir, "Longitudinal hadronic flow at RHIC in extended statistical thermal model and resonance decay effects," Acta Physica Polonica B, vol. 41, no. 11, pp. 2433-2448, 2010.

[11] C. Ristea, A. Jipa, I. Lazanu et al., "Hubble flow in relativistic heavy ion collisions," Journal of Physics: Conference Series, vol. 420, no. 1, Article ID 012040, 2013.

[12] S. Uddin, N. Akhttar, and M. Ali, "Pion production and collective flow effects in intermediate energy nucleus-nucleus collisions," International Journal of Modern Physics A, vol. 21, no. 7, p. 1471, 2006.
[13] B. Abelev, J. Adam, and D. Adamová, "Multi-strange baryon production at mid-rapidity in $\mathrm{Pb}-\mathrm{Pb}$ collisions at $\sqrt{\mathbf{s}_{\mathrm{NN}}}=$ 2.76 TeV," Physics Letters B, vol. 728, pp. 216-227, 2013.

[14] Iouri Belikov (for the ALICE Collaboration), " $K^{0}{ }_{s}$ and $\Lambda$ production in $\mathrm{Pb}-\mathrm{Pb}$ collisions with the ALICE experiment," Journal of Physics G: Nuclear and Particle Physics, vol. 3, no. 12, Article ID 124078, 2011.

[15] B. Abelev, J. Adam, and D. Adamová, "Centrality dependence of $\pi, K$, and p production in $\mathrm{Pb}-\mathrm{Pb}$ collisions at $\sqrt{\mathbf{s}_{\mathrm{NN}}}=2.76 \mathrm{TeV}$," Physical Review C, vol. 88, Article ID 044910, 2013.

[16] P. Huovinen and P. V. Ruuskanen, "Hydrodynamic models for heavy ion collisions," Annual Review of Nuclear and Particle Science, vol. 56, pp. 163-206, 2006.

[17] K. J. Eskola, H. Honkanen, H. Niemi, P. V. Ruuskanen, and S. S. Rasanen, "Hadron multiplicities, pT-spectra and netbaryon number in central $\mathrm{Pb}+\mathrm{Pb}$ collisions at the LHC," http:// arxiv.org/abs/0705.1770.

[18] M. Rybczynski, W. Florkowski, and W. Broniowski, "Singlefreeze-out model for ultrarelativistic heavy-ion collisions at $\sqrt{\mathbf{s}_{\mathrm{NN}}}=2.76 \mathrm{TeV}$,' Physical Review C, vol. 85, no. 5, Article ID 054907, 9 pages, 2012. 

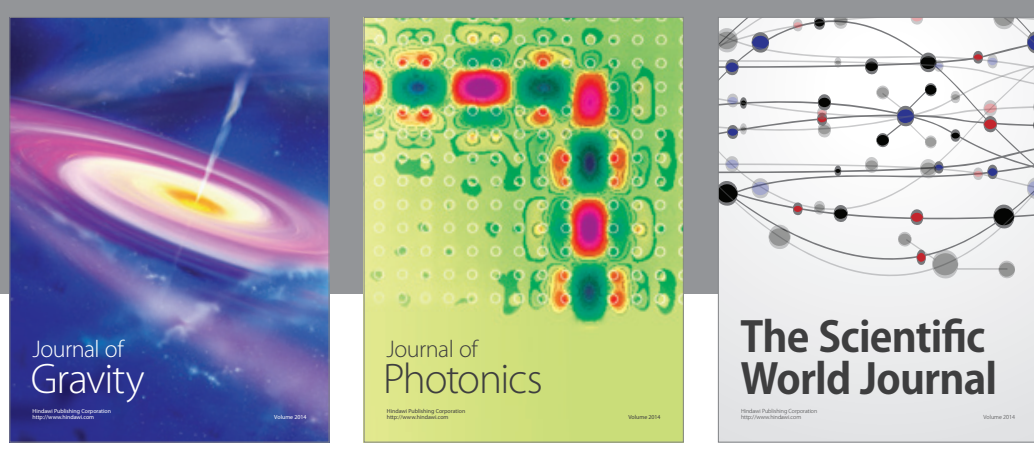

The Scientific World Journal


Submit your manuscripts at

http://www.hindawi.com

nternational Journal of

Statistical Mechanics
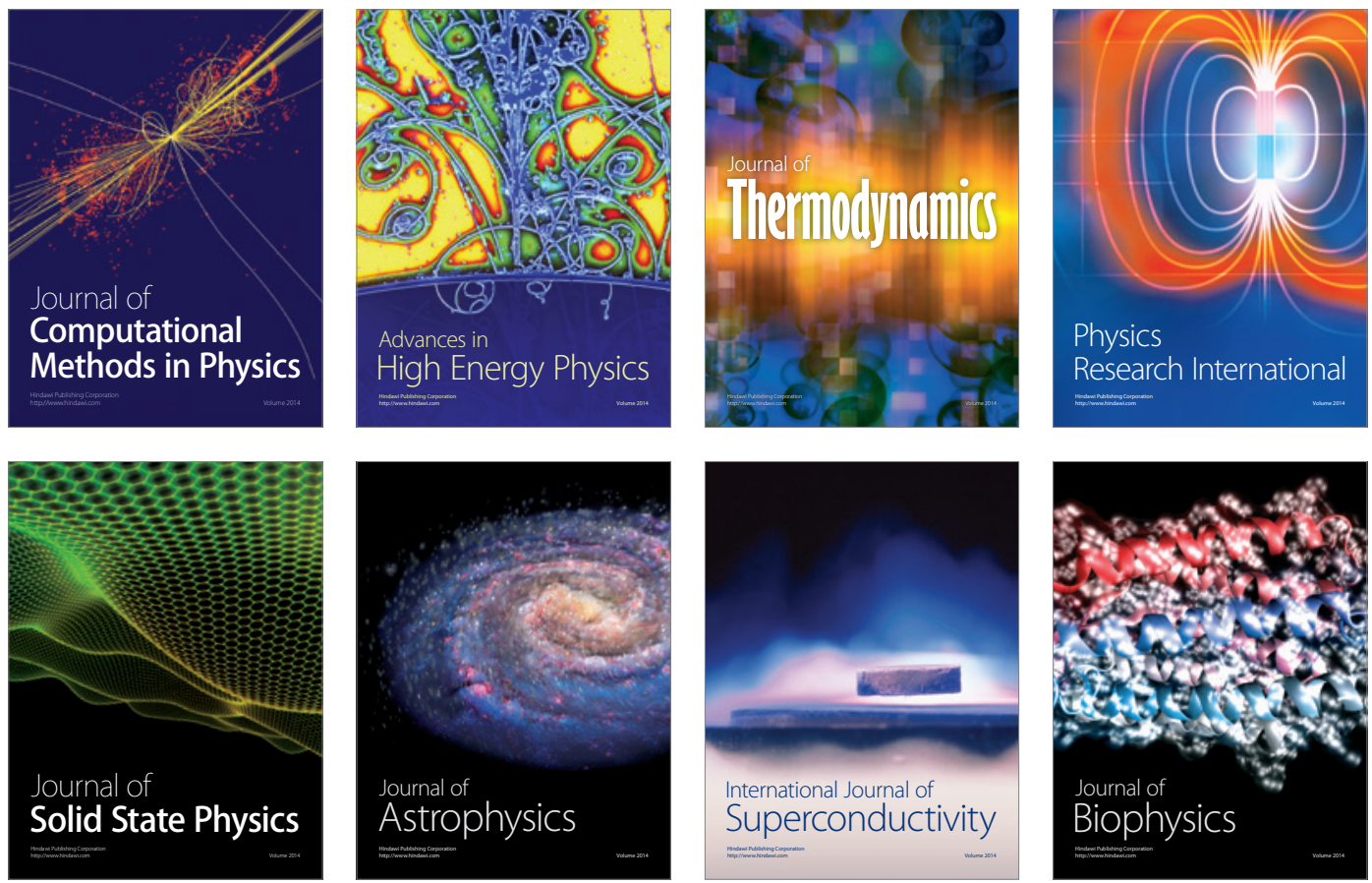
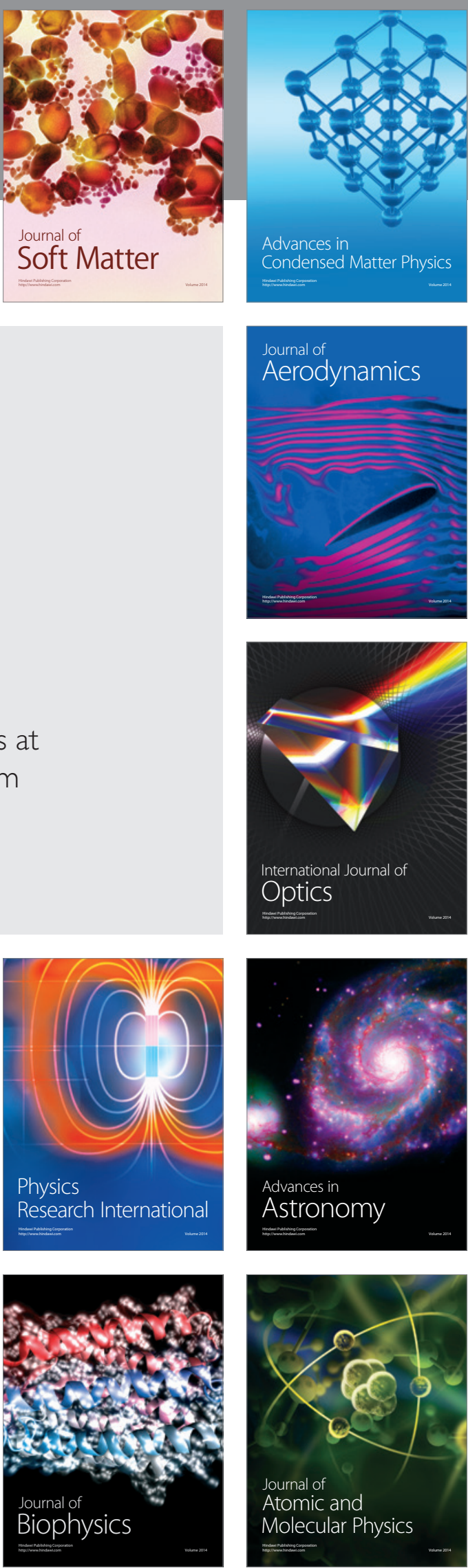